\title{
Anandamide activation of CB1 receptors increases spontaneous bursting and oscillatory activity in the thalamus
}

\author{
M. Dasilva, K.L. Grieve, J. Cudeiro, C. Rivadulla
}

\begin{abstract}
The endocannabinoid system is a modulatory system that has been strongly associated with the regulation of functions as learning and memory, pain perception and sensory physiology in many areas of the central nervous system. However, although a role in sensory processing has been demonstrated at the level of the thalamus, the influence of the endocannabinoid system on thalamic rhythms and oscillations has been less studied, despite the fact that such activities are significant characteristics of the thalamic state.

The present work aimed to characterize the role of anandamide (AEA) - one of the endogenous CB1 receptor agonists - and AM251 - a CB1 antagonist - in the modulation of burst firing and oscillatory activity present in the dLGN of the anesthetized rat. Administration of AEA $(0.5 \mathrm{mg} / \mathrm{kg}$ iv $)$ increased the number of bursts in the majority of the cells tested and induced the appearance of a slow delta-like $(1.5 \mathrm{~Hz})$ oscillatory activity. These effects were CB1-mediated, as demonstrated by the complete antagonism during the co-application of AM251 (0.5 mg/kg iv). Thus, by demonstrating that the AEA-mediated activation of CB1 receptors increases spontaneous bursting and oscillatory activity in the thalamus our study infers that endocannabinoids could have a role in processes controlling the sleep-wake cycle and level of arousal.
\end{abstract}

Key words: endocannabinoids; thalamus; anandamide; bursts; oscillations; sleep

\section{Introduction}

The endocannabinoid anandamide (AEA) is an arachidonic acid derivative (Devane et al., 1992 and Mechoulam et al., 1995) which acts via two families of G-protein-coupled receptors, CB1 and CB2 (Matsuda et al., 1990 and Munro et al., 1993). As a fatty acid component of membrane phospholipids (Di Marzo et al., 1994) AEA is synthesized and released on demand following a depolarization-induced increase in the intracellular concentration of calcium (Sugiura et al., 1996), which is mainly triggered by the activation of group I metabotropic glutamate receptor (mGluR) and muscarinic acetylcholine receptor (mAChR) receptors (Maejima et al., 2001 and Kim et al., 2002).

The best described action of AEA is the retrograde modulation of neurotransmitter release by an action on presynaptic CB1 receptors (Freund et al., 2003). This has been described mainly in the hippocampus and cerebellum, where AEA has been shown to inhibit both GABAergic and glutamatergic neurotransmission (Schlicker and Kathmann, 2001 and Piomelli, 2003). However, the activation of CB1 receptors by AEA can also increase the release of glutamate (Glu) and acetylcholine (ACh) in the cerebral cortex and hippocampus (Acquas et al., 2000 and Ferraro et al., 2001).

In the somatosensory thalamus, Sun et al. (2011) have recently demonstrated the presence of high levels of CB1 receptors and suggested a role in regulating both inhibitory synapses within the thalamic reticular nucleus (TRN) and excitatory synapses between cortex and thalamus. These results that suggest CB1 receptors are in a unique position to modulate sensory transmission and oscillatory activity within the thalamocorticothalamic circuit. In addition, we have previously demonstrated that application of both locally and systemically applied cannabinoid agents can alter the firing rates of individual visual thalamic cells in the rat, both during driven and spontaneous activities (Dasilva et al., 2012) clearly dividing the population into cells excited and inhibited by cannabinoid activation. In that study, we noted that the level of spontaneous activity appeared to be a factor in the polarity of the effect of exogenous cannabinoids, such that cells excited by the drugs had significantly lower spontaneous activity than those inhibited.

An obvious extension to these studies is to consider how cannabinoids may affect cells interacting as populations, influencing synchronous and rhythmic firing since (i) rhythms are a fundamental part of thalamic activity, (ii) rhythms are mediated at the level of the thalamus by ACh, GABA and Glu, neurotransmitters highly modulated by the endocannabinoid system (Ferraro et al., 2001 and Schlicker and Kathmann, 2001), and (iii) a role for the endocannabinoid system in regulating neuronal rhythmicity 
and synchrony has been previously described (Hájos et al., 2000, Willinsky et al., 1975, Buonamici et al., 1982, Consroe et al., 1997, Ilan et al., 2004, Robbe et al., 2006, Hajós et al., 2008, Morgan et al., 2008, Mason and Cheer, 2009, Sun et al., 2011 and Sales-Carbonell et al., 2013).

Thalamic neurons show oscillatory activity in a broad range of frequencies ranging from slow oscillations $(0.1-1 \mathrm{~Hz})$ during deep non-paradoxical sleep, to gamma oscillations $(30-80 \mathrm{~Hz})$ during awareness (Steriade et al., 1993). Further, thalamic cells fire action potentials in two different patterns: (i) tonic spikes and (ii) spikes in burst. Bursts include those spikes that crown a calcium action potential generated by the activation of a low-threshold calcium current preceded by a hyperpolarized state (Jahnsen and Llinás, 1984 and Lu et al., 1992), and have been classically associated to sleep-like states (Livingstone and Hubel, 1981 and Steriade et al., 1993).

Here we examine the effects of systemically applied AEA and AM251 -a CB1 receptor agonist and antagonist, respectively- focussing on two important and related aspects of thalamic function - the rhythmic firing pattern found when thalamic cells fire in burst mode and sleep-like oscillatory activity (Steriade et al., 1993).

\section{Experimental procedures}

\section{Animals and preparation}

Experiments were carried out in six Lister-hooded adult rats weighing between 250 and $350 \mathrm{~g}$. All procedures complied with the ethical use of animals specified in the European Communities Council Directive of November 24, 1986 (86/609/EEC) and Animals (Scientific Procedures) Act 1986, UK Home Office.

Anesthesia was induced with a mixture of ketamine $\left(50 \mathrm{mg} \mathrm{kg}^{-1}\right.$, Ketolar, Parke-Davis, Madrid, Spain) and xylazine (10 $\mathrm{mg} \mathrm{kg}^{-1}$, Sigma-Aldrich, Madrid, Spain) given intra-peritoneally, while an intravenous mixture of ketamine $\left(13 \mathrm{mg} \mathrm{kg}^{-1} \mathrm{~h}^{-1}\right)$ and xylazine $\left(1.5 \mathrm{mg} \mathrm{kg}^{-1} \mathrm{~h}^{-1}\right)$ in glucose saline was supplied through the lateral tail vein for surgery and maintenance via an infusion pump (Harvard Apparatus).

The ECG was continuously observed (Biopac Systems Inc., CA, USA) and maintained within normal physiological limits to ensure an appropriate level of anesthesia. Body temperature was also continuously measured and kept around $37 \pm 0.5^{\circ} \mathrm{C}$ using a thermal blanket (Harvard Apparatus Homeothermic Blanket). Lidocaine gel was applied to pressure points.

Animals were placed in a customized stereotaxic apparatus such that the head was secured in a horizontal orientation and the entire right visual hemifield was unobstructed. To access the dorsal lateral geniculate nucleus (dLGN), the skull was exposed, a craniotomy was drilled $4.5 \mathrm{~mm}$ posterior to bregma and $3.5 \mathrm{~mm}$ lateral to the midline (Paxinos and Watson, 2007), and the dura mater was retracted with a curved needle. After experiments animals were killed with an anesthetic overdose without recovering from anesthesia.

\section{Recording of single neuronal activity and electrocorticographic (ECoG)}

A hydraulic microelectrode multidrive of eight channels (tungsten electrodes, 1.0 M $\Omega$, FHC Inc., ME, USA) was used to record simultaneously the extracellular activity of groups of dLGN neurons during spontaneous, undriven activity. To locate the dLGN, the multidrive was positioned over the craniotomy and each single microelectrode was advanced into the brain while stimulating the appropriate eye with hand-held stimuli. After reaching the dLGN, extracellular single-cell neural signals with a good signal-tonoise ratio were isolated and continuously monitored using a 310 Tektronix Oscilloscope (Tektronix Holland N.V., Heerenveen, The Netherlands) and an audio monitor (FHC Inc.). These signals were preamplified (Preamplifier board, Plexon Inc., TX, USA) and fed into a multichannel acquisition processor (Plexon, Inc.) that allowed the amplification, filtering and real-time spike sorting of the acquired signals, which were stored in a computer provided with RASPUTIN software (Real-time Acquisition System Programs for Unit Timing in Neuroscience, Plexon Inc.). Off-line analyses were carried out using Offline Sorter and Neuroexplorer softwares (Plexon Inc.), MS Excel (Microsoft Corporation), MATLAB (The MathWorks Inc., MA, USA) and SPSS (SPSS Inc., NY, USA). ECoG activity was recorded by means of a parallel bipolar microelectrode (FHC Inc.) positioned in the left visual cortex. Activity was filtered $(0.1 \mathrm{~Hz}-1 \mathrm{kHz})$ and preamplified using a differential AC amplifier (AM Systems, WA, USA) and fed into a continuous channel of a multichannel acquisition processor (Plexon Inc.). Offline analysis was carried out using Neuroexplorer software (Plexon Inc.). 


\section{Pharmacology}

AEA (N-(2-hydroxyethyl)-5Z,8Z,11Z,14Z-eicosatetraenamide) and AM251 (N-(piperidin-1-yl)-5-(4iodophenyl)-1-(2,4-dichlorophenyl)-4-methyl-1H-pyrazole-3-carboxamide) were obtained from Tocris (Bristol, United Kingdom). Both were dissolved in vehicle (1:1:18 cremophor/ethanol/saline) and applied intravenously via the lateral tail vein using the same concentration $(0.5 \mathrm{mg} / \mathrm{kg})$ and protocol described in our previous work (Dasilva et al., 2012). Once dLGN cells were isolated, spontaneous activity was recorded in control conditions for at least 5 min after which AEA was injected and neuronal activity recorded for a further $10-15 \mathrm{~min}$ to determine the period of maximal effect and recovery. In the experiments where AM251 was used we waited 5 min after AM251 injection before administration of AEA.

\section{Data analysis}

Drug-induced changes were measured by averaging the firing rate over a time window of 5 min at the time of maximal effect after drug administration and comparing this to that obtained using the same duration of time window during the control, pre-drug period. Only those effects where $p<0.05$ were considered significant (one-way analysis of variance (ANOVA) or Friedman tests followed by Dunnett $t$ test or Wilcoxon signed rank test when appropriate). Population data were analyzed using a one-way ANOVA, Friedman test, paired Student $t$-test, Mann-Whitney test or Wilcoxon signed rank test, where appropriate. When necessary, post hoc comparisons were made. Burst analysis was carried out using Matlab software (The MathWorks Inc.). The criteria for considering a train of action potentials as a burst was the presence of two or more spikes with a preceding silent period of at least $50 \mathrm{~ms}$ and an interspike interval not greater than $4 \mathrm{~ms}$ ( Guido et al., 1992; Lu et al., 1992). Action potentials not fulfilling this criterion were considered as tonic firing. The calculation of the number of bursts was achieved by counting how many bursts were within a period of interest, and the percentage of spikes in bursts was defined as the ratio of the number of spikes within bursts to the total number of spikes within that period.

Autocorrelogram and power spectral density (PSD) analyses were carried out using Neuroexplorer software (Plexon Inc.). An autocorrelogram can be defined as a cross-correlogram of a neuronal signal with itself indicating similarities in the firing pattern as a function of time. Thus, in this study, autocorrelograms were used to indicate periodicities and harmonic frequencies in the firing of neurons across a \pm 2 -s time window. The PSDs for individual neurons were calculated across the same 5-min time windows described above. The PSDs of the electrocorticograms were calculated in the same way as for individual neurons, but continuous values in millivolts were used instead of a rate histogram.

\section{Results}

We report the effect of AEA application on 84 neurons in the dLGN of six rats. Up to eight cells were recorded simultaneously, and their activity was studied both in control conditions and during the systemic iv administration of the CB1 receptor agonist AEA $(0.5 \mathrm{mg} / \mathrm{kg})$ and the CB1 antagonist AM251 $(0.5 \mathrm{mg} / \mathrm{kg})$.

\section{CB1 receptor activation alters the spontaneous bursting activity of $d L G N$ relay neurons}

Administration of AEA had a significant effect on the number of bursts compared to the control values (Student $t$-test, $p<0.001, N=84)$. Post-hoc observation showed that the administration of AEA increased the number of bursts in $70 \%$ (59/84) of our population of neurons, and decreased the number of bursts in the other 30\% (25/84) ( Fig. 1). In each case the change in the level of bursts exceeded $10 \%$ of the control values, no cell was unaffected, and the effect lasted on average $5 \mathrm{~min}$, thereafter returning to control levels. Each of these effects was found to be CB1-specific, as the effects were completely blocked during the subsequent co-administration of AM251 and AEA in a sub-set of 36 cells (22 increased the number of bursts in the presence of AEA alone and 14 decreased the number of bursts; Fig. 1).Those cells which elevated their burst firing in response to AEA ( Fig. 1a, upper histogram) did not change their overall firing rate ( Fig. 1a lower histogram) while those which showed a decrease in the number of bursts ( Fig. $1 \mathrm{~b}$, upper histogram) showed a significant decrease in spontaneous firing ( Fig. 1b, lower histogram). However, in the control conditions these two groups were not significantly different $(p>0.05$, MannWhitney $U$ test). The administration of AM251 alone $(N=42$, Fig. $1 \mathrm{c})$ or vehicle alone $(N=34$, data not shown) had no significant effects ( $p>0.05$ vs. control, Wilcoxon signed-rank test). An example of the firing patterns of three simultaneously recorded individual cells is shown in the raster plots in Fig. 1d. The 
clear increase in bursting activity during AEA application is seen in the lower recordings (a $48 \%$ increase on average for these cells), with the development of an oscillatory pattern (see below).

a

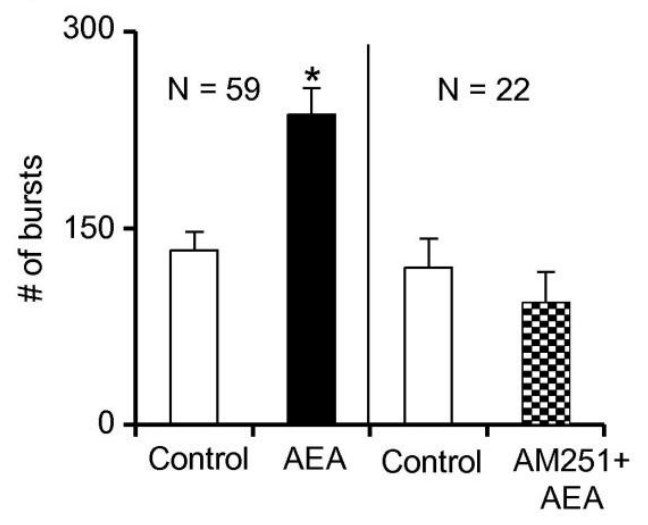

Spontaneous activity

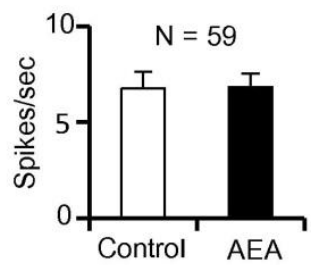

C

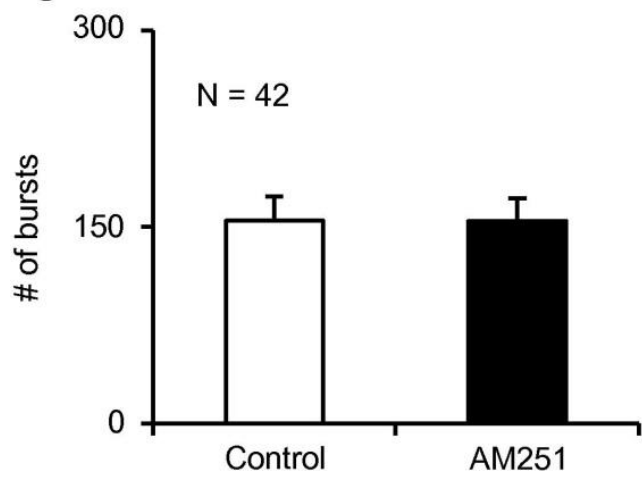

b

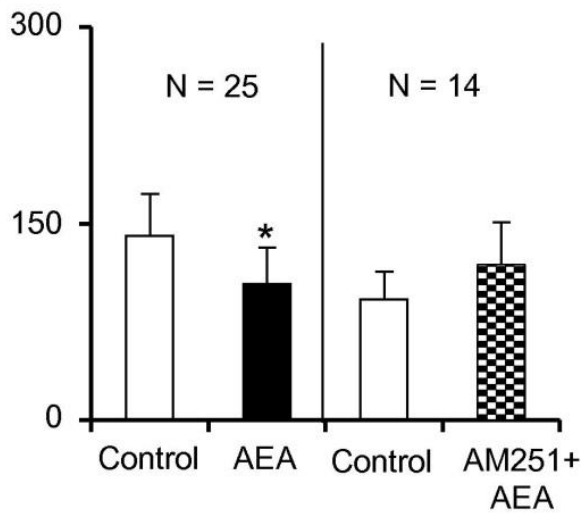

Spontaneous activity

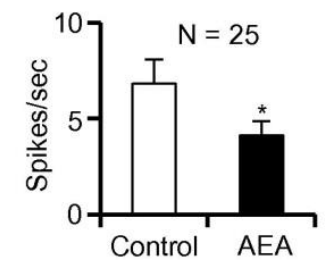

d

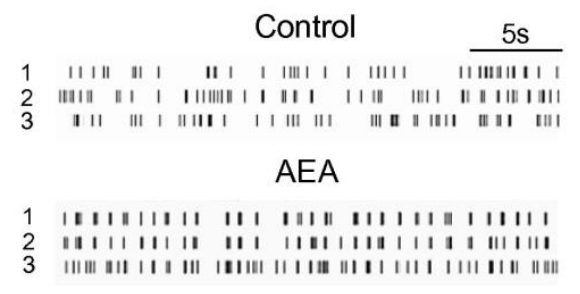

AEA

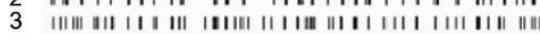

Fig. 1. Effect of AEA on the rate of bursting of dLGN neurons during spontaneous activity. (a, b) Upper figures: bar histograms represent the average number of bursts observed in the population during $5 \mathrm{~min}$ of spontaneous activity. The administration of AEA $(0.5 \mathrm{mg} / \mathrm{kg}$ iv) had two distinct and different effects in our population of neurons: increasing $(\mathrm{a}, N=59)$ or decreasing (b, $N=25)$ the rate of burst occurrence during spontaneous activity. ${ }^{*} p<0.05$ vs. control, paired Student $t$-test. Further, these effects were abolished by the co-administration of AM251 $(0.5 \mathrm{mg} / \mathrm{kg}$ iv) $(\mathrm{a}, N=22 ; \mathrm{b}, N=14 ; p>0.05 \mathrm{vs}$. control, paired Student $t$-test). Lower figures: bar histograms of the actual firing rates in spikes/s, divided into two groups according to the effect of AEA on the number of bursts fired (top row). Only the second group showed a significant change, a decrease in spontaneous activity ( $p<0.05$ vs. control, paired Student $t$-test). (c) Bar histogram representing the lack of effect of AM251 on the number of bursts when applied alone. (d) Representative spike raster plots for three different neurons recorded simultaneously, showing the activity during control condition (upper panel) and during the induction of burst firing by the administration of AEA (lower panel). All data are expressed as mean \pm S.E.M.

We found that the cells which increased the number of bursts during the administration of AEA also increased the percentage of spikes in bursts, number of spikes per burst and burst duration (Fig. 2a), while these parameters where not significantly affected in neurons whose burst responses were decreased by AEA (Fig. 2b). Similarly, co-administration of AM251 completely blocked these effects of AEA (Fig. 2). 
a
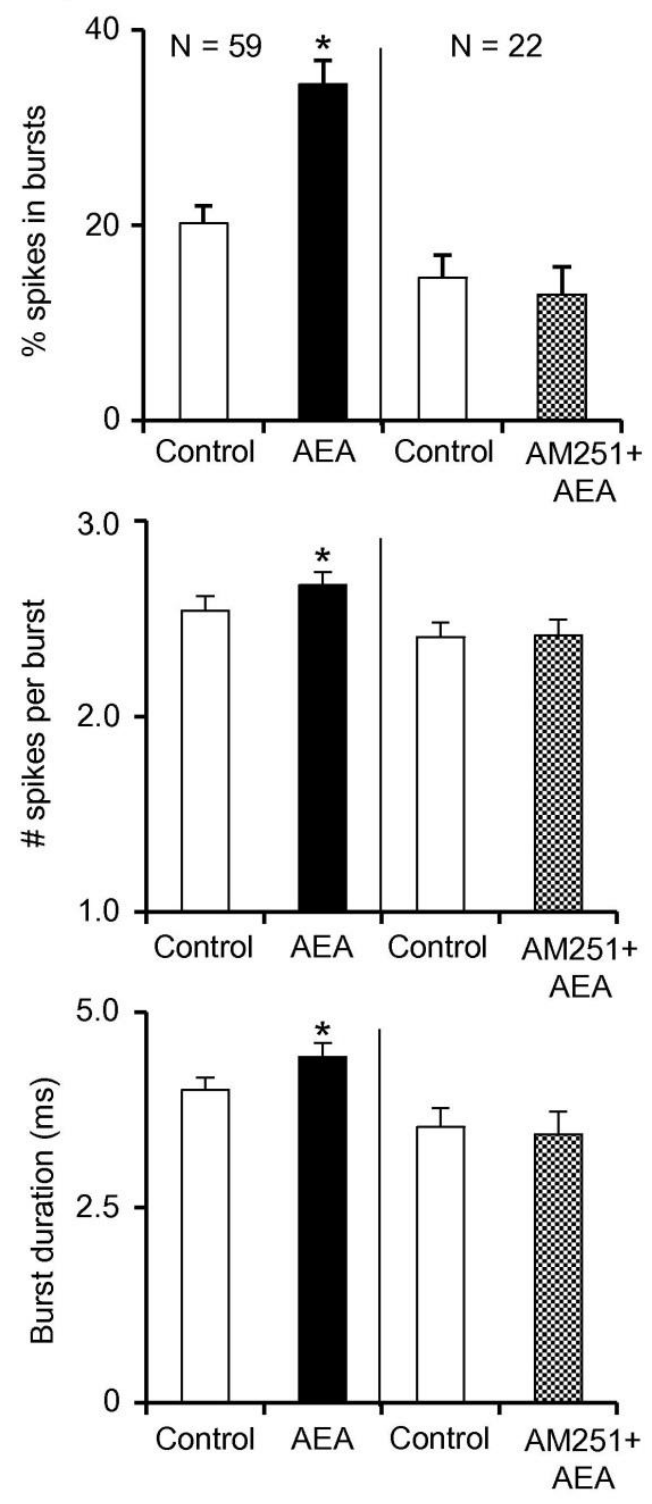

b
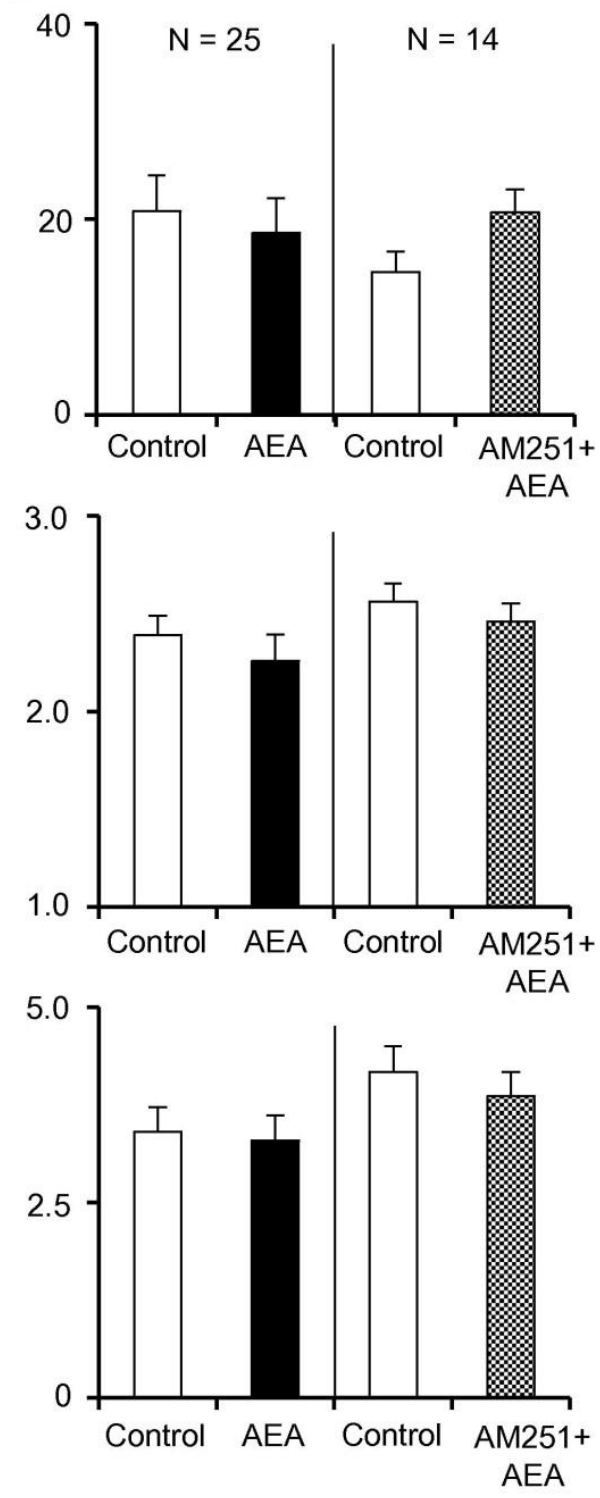

Fig. 2. Effect of AEA ( $0.5 \mathrm{mg} / \mathrm{kg}$ iv) on intrinsic bursts characteristics in dLGN neurons during spontaneous activity. Neurons that increased the number of bursts in the presence of AEA (a) also increased the percentage of spikes in bursts, the number of spikes per burst and burst duration. Neurons where AEA induced a decrease in the number of bursts (b) did not show any change in any of these parameters (a, $N=59 ; \mathrm{b}, N=25$ ). ${ }^{*} p<0.05$ vs. control, paired Student $t$-test. Further, the administration of the CB1 antagonist AM251 $(0.5 \mathrm{mg} / \mathrm{kg}$ iv) to a subpopulation of cells totally blocked the AEA-mediated effects on intrinsic burst characteristics (a, $N=22 ; \mathrm{b}, N=14), p>0.05$ vs. their own control, paired Student $t$-test. Data expressed as mean \pm S.E.M.

\section{AEA induces a delta-like oscillatory activity pattern in the dLGN}

We investigated the effect of AEA on the oscillatory activity pattern of our population of neurons finding that it induced a delta-like oscillation of $1.5 \mathrm{~Hz}$ in a significant majority of the cells tested $(N=59,70 \%)$. This effect is illustrated in Fig. 3, which shows the autocorrelograms of six simultaneously recorded dLGN neurons in control condition (a) and during the administration of AEA (b), where all began to oscillate at delta-like frequencies. Interestingly, we found a positive correlation between the appearance of this AEA-mediated delta-like oscillation and the increase in burst firing previously described. The development of an oscillatory pattern is clear even by simple visual inspection in Fig. 1d, during AEA administration. Specifically, those neurons that had increased the number of bursts during the presence of AEA (59/84, see above) shifted into oscillation at $\sim 1.5 \mathrm{~Hz}$ ( Fig. 4a black line, Fig. 4c), while the pattern of those neurons that had decreased their burst firing (25/84) was not significantly altered ( Fig. 4b black line, Fig. 4c). Furthermore, it can be seen by comparing Fig. 4a and b that these two groups of neurons 
also differed in their oscillatory activity under control conditions, prior to drug administration. Those neurons that started to oscillate at delta-like frequencies in the presence of AEA were already oscillating at slow-like frequencies $(0.5 \mathrm{~Hz})$ in control conditions ( Fig. 4 a solid gray line), while those neurons that did not change their oscillatory activity in the presence of AEA (25/84) did not oscillate at this frequency ( Fig. 4b solid gray line). Fig. 4d shows an example of two simultaneously recorded cells, representing on the left a cell that showed a slow-like frequency in control conditions that switched to $1.5 \mathrm{~Hz}$ with the administration of AEA, while the cell shown on the right did not oscillate in control conditions or during drug application. These results suggest that the observed effects are not the consequence of a global change in state, as the two cells reported above were recorded simultaneously and yet had opposite responses to drug administration.

a
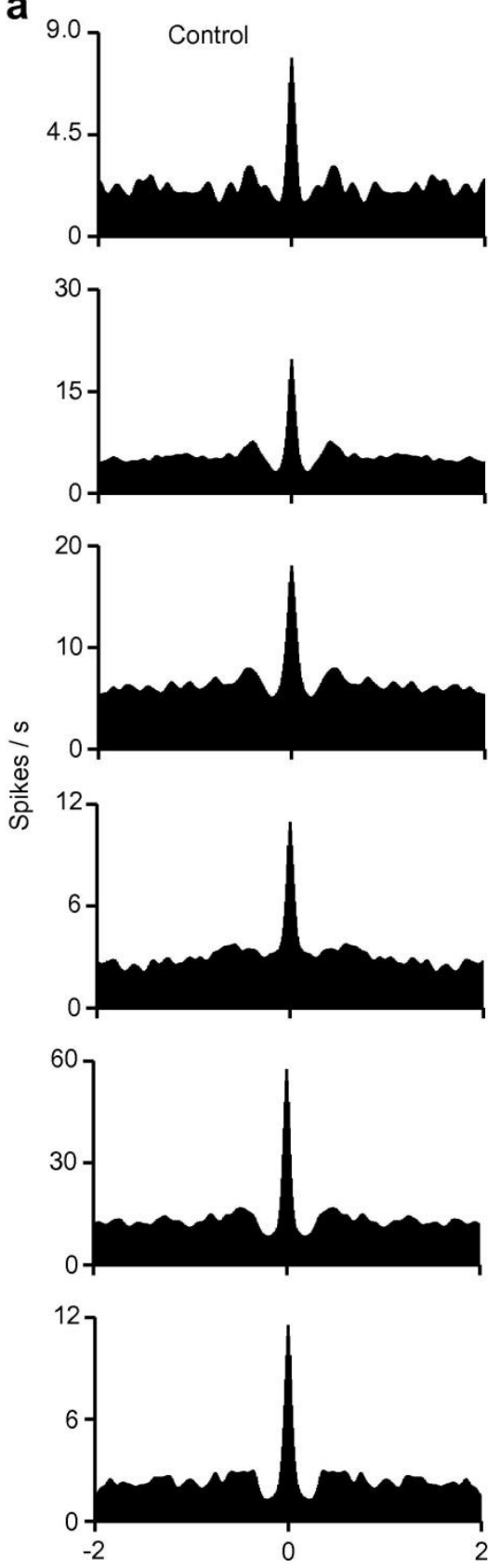

b
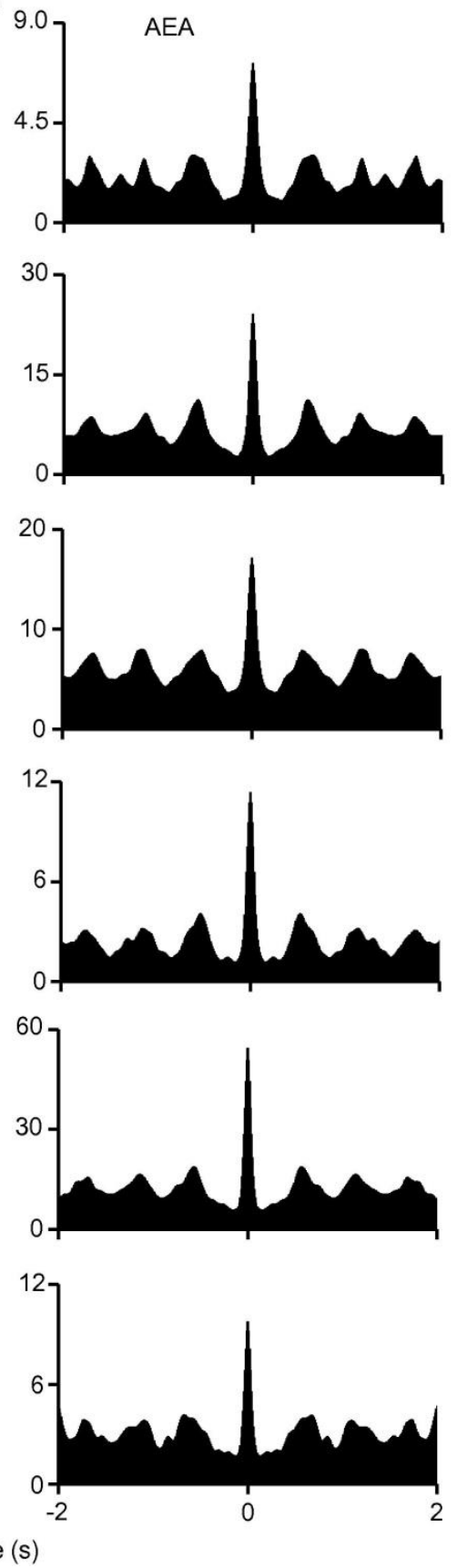

Fig. 3. Autocorrelograms of six simultaneously recorded dLGN neurons during control condition (left) and presence of AEA $(0.5 \mathrm{mg} / \mathrm{kg}$ iv, right). The administration of AEA induced an oscillatory activity at delta-like frequencies $(1.5 \mathrm{~Hz})$ in all the cells. 

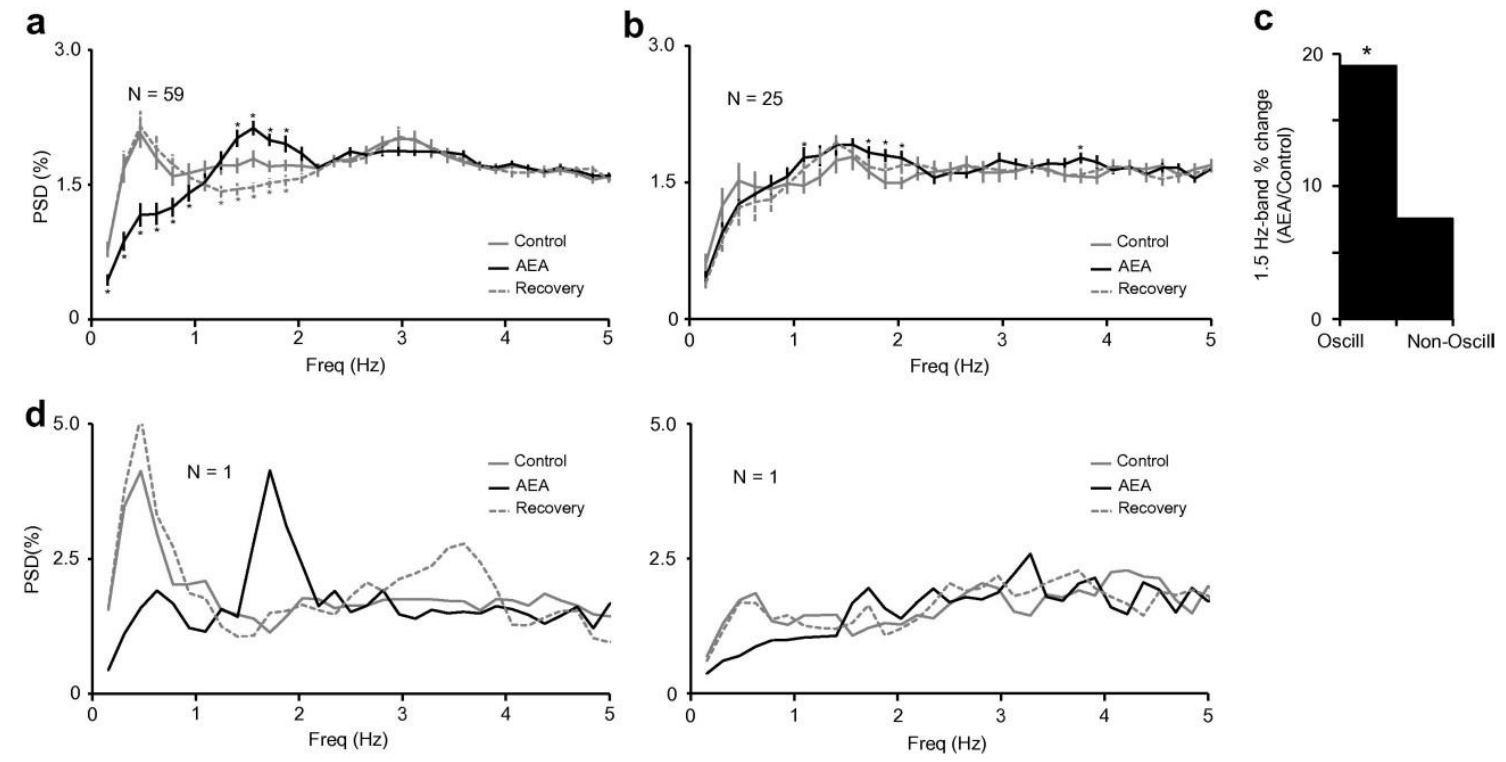

Fig. 4. PSD graphs showing the effect of AEA on the oscillatory activity patterns of the neurons that increased (a) or decreased (b) their rate of bursting. (c) Bar histogram comparing the percentage of increase in the $1.5-\mathrm{Hz}$ band vs. control, for the two populations. (d) The power spectrum analysis of two simultaneously recorded cells showing that the cell (left) previously oscillating changes the frequency under AEA influence, while the cell (right) that is not oscillating in control is not induced to do it by the application of the drug. ${ }^{*} p<0.05$ vs. control, Wilcoxon signed-rank test. Data are expressed as mean \pm S.E.M.

Since we recorded multiple single units simultaneously, in groups ranging from four to eight cells, in several animals, the simplest way to view this data is as shown in Fig. 5, showing the entire set of 84 cells. Here each bar represents the number of cells recorded simultaneously from a single eight electrode array, which was reinserted up to three times in each of the six animals. White bars indicate the number of simultaneously recorded cells which increased bursting and black ones those which decreased. The obvious scattering in some penetrations/animals, versus the complete single effect in others does not permit a clean suggestion of a single effect based upon the animals status at the time, rather there are multiple effects seen across populations of cells. Further, since these electrodes were stereotaxically placed vertically into the dLGN, there is also no obvious internal location effect or apparent laminar effect. 


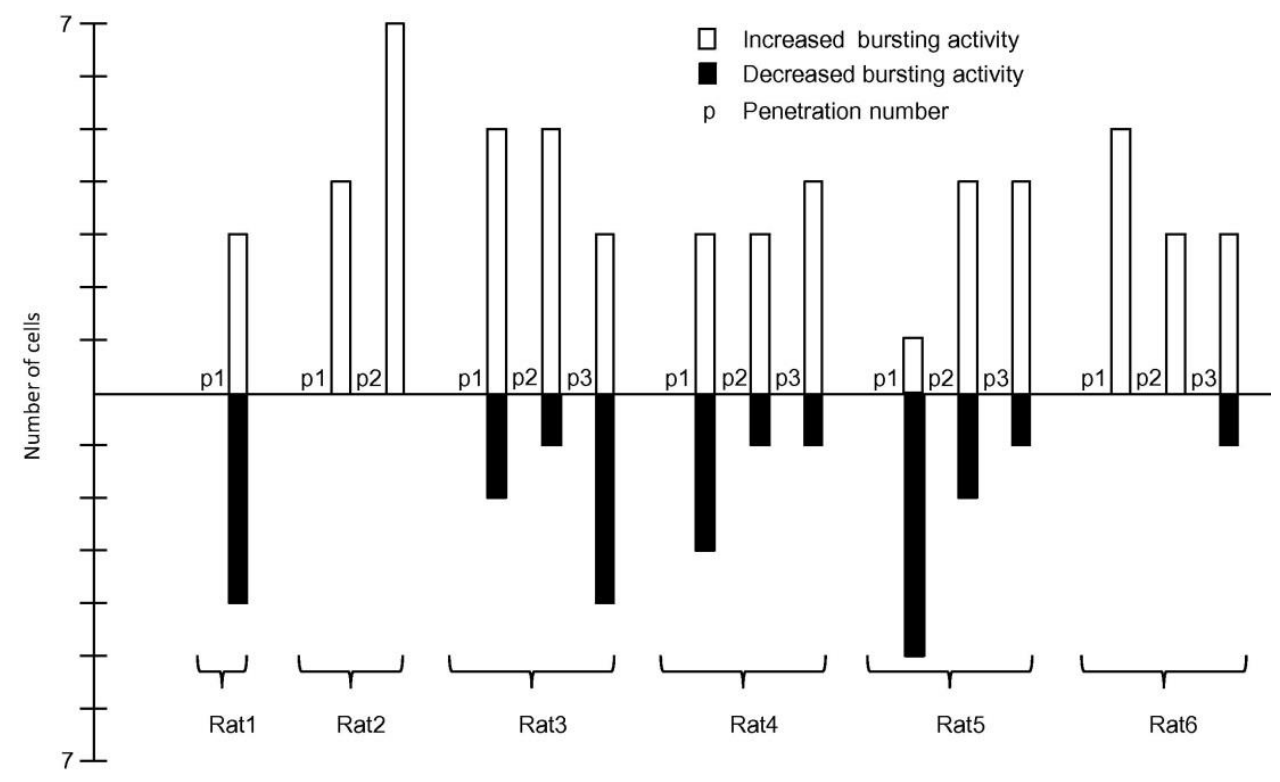

Fig. 5. Each bar represents the number of cells recorded simultaneously in a single penetration (up to eight electrodes), the white portion indicates the number of cells which increased bursting, and the black portion those cells that decreased bursting after AEA application. While all cells recorded in animal 2 and almost all in animal 6 showed increased bursting in response to AEA, there was a much more mixed picture overall, and no suggestion of a physical or temporal pattern.

Co-administration of AM251 to a subgroup of cells oscillating at $1.5 \mathrm{~Hz}$ as a result of AEA injection $(N=19)$ completely abolished the oscillation. This effect is exemplified in Fig. 6, which shows the activity of one of these cells during control condition (left), during the presence of AEA (middle) and coadministration of AM251 (right). Further, administration of AM251 alone was without effect $(N=8$, Fig. $7 \mathrm{a})$, as was injection of vehicle alone $(N=13$, Fig. $7 \mathrm{~b})$.
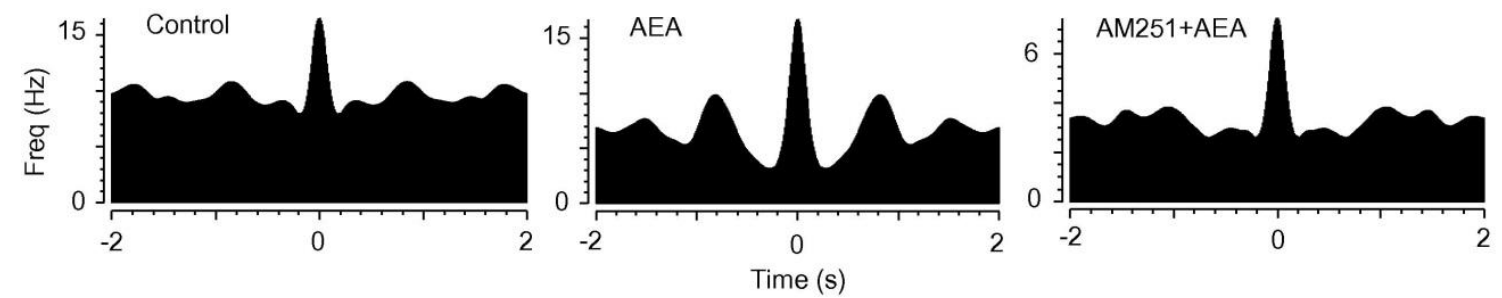

Fig. 6. Autocorrelograms of a single dLGN neuron during control (left), application of AEA ( $0.5 \mathrm{mg} / \mathrm{kg}$ iv, middle) and coadministration of AEA and AM251 (0.5 mg/kg iv, right). The slow delta-like oscillation induced by the presence of AEA (middle) is totally blocked when the CB1 antagonist AM251 is co-applied (right). 

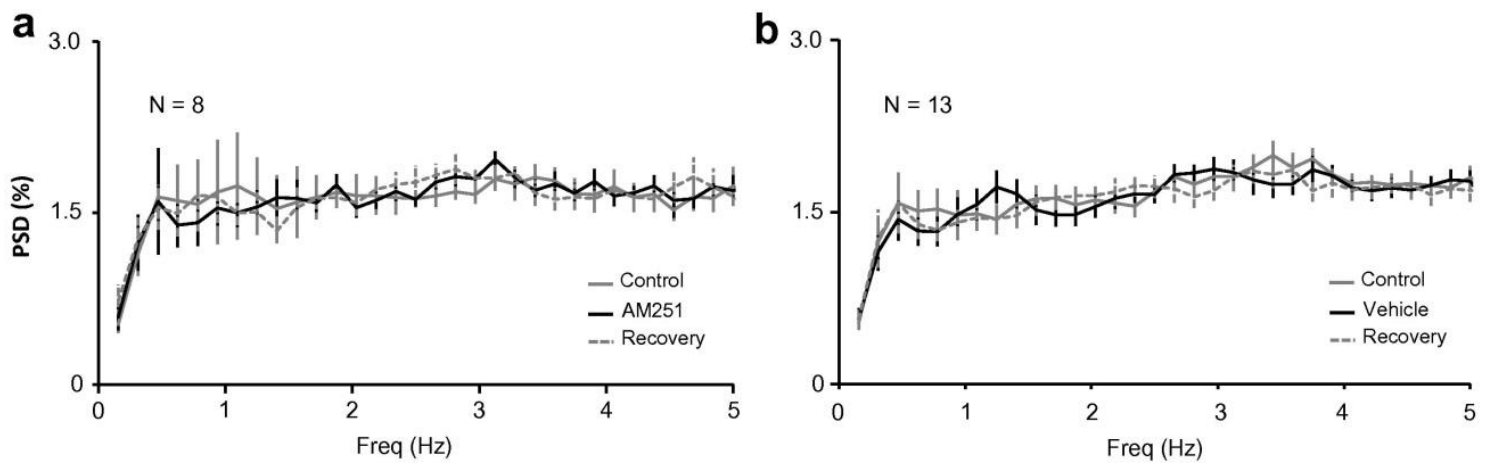

Fig. 7. Psd graphs averaging the effect of AM251 (a) and vehicle (b) when administered alone to a subgroup of cells where the presence of AEA induced the appearance of a $1.5-\mathrm{Hz}$ oscillation. The population data are expressed as mean \pm S.E.M. No significant effects were detected in any case ( $p>0.05$ vs. control, Wilcoxon signed-rank test).

The delta-like thalamic oscillation induced by AEA administration was also seen at the level of the cerebral cortex. This is shown in Fig. 8, which shows an ECoG recorded from V1 during control conditions (a), presence of AEA (b) and recovery (c). AEA administration clearly reinforced the power of a previously weak delta-like oscillation around $2.0 \mathrm{~Hz}$, while it totally suppressed the $0.5-\mathrm{Hz}$ slow-like activity also present during control conditions. These effects were also seen at the population level, as shown in Fig. $8 \mathrm{~d}$ and e, which illustrate the power of the slow-like $(0-0.5 \mathrm{~Hz})$ and delta-like $(1-2 \mathrm{~Hz})$ bands respectively, during control condition (left column), presence of AEA (middle column) and recovery (right column). While the delta-like oscillatory activity was significantly increased, the slow-like activity was decreased.

a

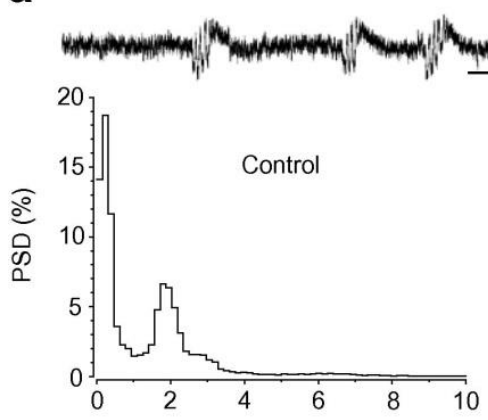

b

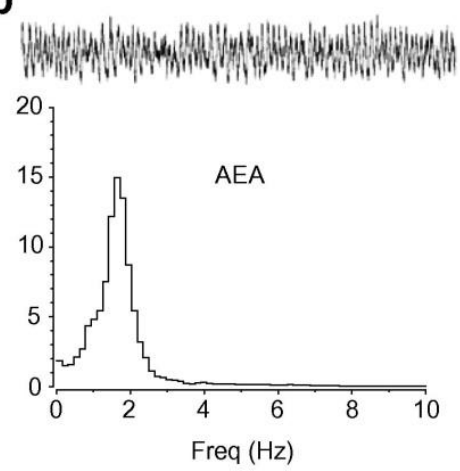

C

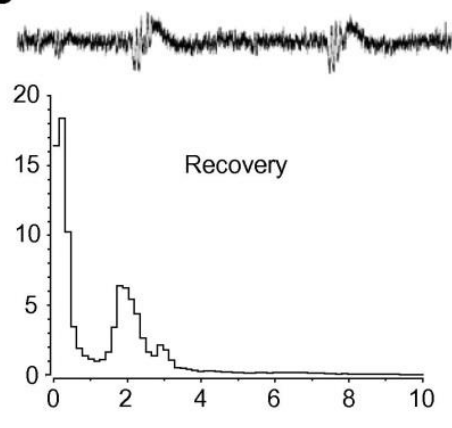

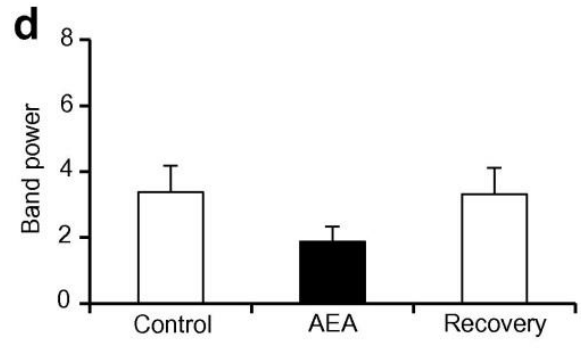

Slow-like activity

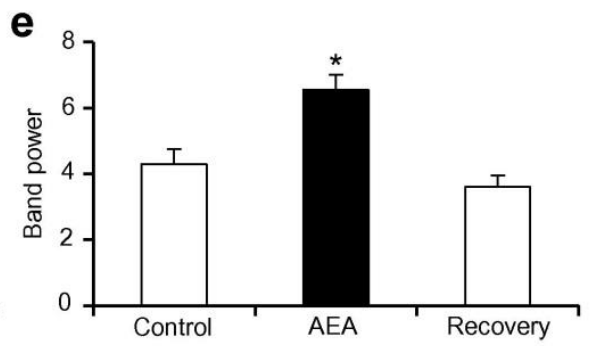

Delta-like activity

Fig. 8. The effects induced by AEA were also reflected at the ECoG level. A raw example of an ECoG is shown (upper traces) during control (a), administration of AEA (b) and recovery (c), along with the corresponding PSD analysis for each condition. In this example, AEA clearly increased the power of the oscillatory activity in the delta range while abolishing a slow-like oscillation present during control conditions. (Scale bars $=1 \mathrm{mV}$ and $5 \mathrm{~s}$ ). (d, e) show the mean data for the population $(N=11)$. ${ }^{*} p<0.01 \mathrm{vs}$. control, Friedman test with Bonferroni correction. 


\section{Discussion}

Our data suggest that our pharmacological alteration of the endocannabinoid system during a physiological steady-state induces a significant shift in the firing pattern of thalamic cells. This activation of the CB1 receptor-mediated "network" results in a significant increase in burst firing in a majority of the cell population, including changes in burst duration and the number of spikes involved, all indicative of enhanced inhibition or withdrawal of an excitatory drive, resulting in hyperpolarization. However, we also found a smaller but significant proportion of cells which did not follow this pattern. These cells showed a small decrease in some elements of burst firing, but the other burst measures were unchanged. Interestingly, there was no overall shift in the basal firing rate of those cells which increased burst firing, but a significant decrease in those in which the number of bursts fired was reduced, which may again suggest increased inhibition or decreased excitation. However, it is known that the relationship between the degree of inhibition and the level of burstiness is complex, particularly in vivo (Grieve et al., 2009).

More interestingly, the firing pattern measured both at the single-cell level, and at the higher level, in terms of thalamocortical interactions revealed via the ECoG, have shown a shift in oscillatory patterned activity with a significant shift from frequencies below $1 \mathrm{~Hz}$ to a peak at $1.5 \mathrm{~Hz}$ in the delta range. Crucially, this only occurred in those cells which had shown an increase in burst firing. Moreover, cells which did not show this shift did not show a significant low-frequency oscillation in control conditions.

These two response types were found intermingled in groups of cells recorded simultaneously (Fig. 5) indicating that our pharmacological intervention did not simply shift the thalamic "state", rather one population of cells shifted to a burstier pattern with more $1.5-\mathrm{Hz}$ activity, while a second population reduced spontaneous firing, with no major shift in oscillation. There was no evidence of specific thalamic locations or laminae showing distinct effects, the two seemed to be almost randomly distributed with no obvious groupings. Since we elected to examine only spontaneous activity in these cells, we have no further evidence to suggest division of these populations by physiological sub-type.

The two main effects described here (increase of bursting activity and induction of delta-like oscillations) seem to be the consequence of the hyperpolarization of thalamic cells due to the activation of $\mathrm{CB} 1$ receptors. Indeed, the increase in burst firing in these cells is a landmark of a general hyperpolarized state in the dLGN (Sherman and Guillery, 2002) and is in agreement with the reported inhibitory effect of CB1 activation during visual stimulation in the dLGN of the rat (Dasilva et al., 2012). In addition, this assumption is supported by the fact that delta oscillations are characteristic of thalamocortical cells during deep non-REM (Rapid Eye Movement) sleep (Steriade et al., 1993), a state in which thalamocortical relay neurons are hyperpolarized (Steriade et al., 1993 and Steriade, 2004) and start to oscillate intrinsically at delta frequencies, due to the interplay of $I_{\mathrm{h}}$ and $I_{\mathrm{t}}$ currents ( Dossi et al., 1992). Interestingly, application of $\mathrm{CB}$ receptor antagonist, while completely reverted the effect, did not have a measurable effect when tested alone. Although this may suggest that CB mediated basal activity is absent due to our experimental conditions, similar results have been reported in awake rats ( Robbe et al., 2006). Our secondary population, although showing less bursting activity, did show a significant decrease in firing rate, which could also indicate a relative hyperpolarization, although not enough to reach a "bursting state". However, in contrast to our previous study, we did not find any cells with spontaneous activity elevated by the activation of $\mathrm{CB} 1$ receptors, probably due to the use of a different anesthetic regime - chosen to allow a direct comparison with previous studies on thalamocortical oscillations ( Steriade et al., 1993) - and to the study design itself, which called for longer periods of recording in order to maximize the number of bursts recorded, such that small brief elevations might be averaged below significance.

A simple explanation for exogenously applied endocannabinoids to induce a general hyperpolarization in the dLGN would be a dysfacilitation of dLGN neurons by reducing excitatory inputs. One of the main depolarizing afferents to the dLGN is the cholinergic input coming from the brainstem (Steriade, 2004). This has a significant role in the maintenance of arousal and shift from sleep to wake, hence an endocannabinoid-mediated inhibition of this input would effectively hyperpolarize the dLGN, increasing its bursting activity and inducing it to oscillate at delta-like frequencies. Actually, this mechanism has already been proposed to underlie the AEA-mediated induction of sleep (REM and non-REM) in rats (Murillo-Rodríguez et al., 1998 and Murillo-Rodríguez et al., 2001) and is part of the normal process during non-REM sleep, where there is a progressive decrease in the firing rate of cholinergic brainstem thalamic inputs (Steriade et al., 1993). Thus, our data support previous behavioral studies in rats that have reported a sleep-inducing effect of endocannabinoids (Santucci et al., 1996, Murillo-Rodríguez et al., 1998 and Murillo-Rodríguez et al., 2001).

Another mechanism that could explain the hyperpolarizing effect of endocannabinoids in the dLGN, potentially acting in concert with the above, is the reduction of the cortical feedback to the thalamus. The dLGN receives significant feedback connections from the cortex (Sefton et al., 1981 and Garey et al., 1991) which activate group I mGluR1a (McCormick and von Krosigk, 1992). These feedback connections are used to transmit slow oscillatory activity to the thalamus which is generated in the cortex 
during non-REM sleep (Steriade et al., 1993 and Steriade, 2003) in an mGluR1a activation-dependent manner (Hughes et al., 2002). The disruption of these connections has a hyperpolarizing effect on relay cells (Kalil and Chase, 1970 and Waleszczyk et al., 2005), maybe mediated by the action of endocannabinoids as Sun et al. (2011) have demonstrated in the somatosensory system, showing that these compounds decrease the strength of thalamocortical synapses. Reviewing our data, in which the cells that shifted to delta-like oscillation during CB1 receptor activation were those that were already oscillating in control conditions at very low frequencies, we submit that this pattern could also follow a local hyperpolarization of the dLGN by withdrawal or reduction of the descending excitatory drive from the cortex, disrupting the slow oscillatory activity generated in the cortex and transmitted to the thalamus. Actually, it is known that slow oscillations are absent in the thalamus of decorticated animals (Timofeev and Steriade, 1996) and our data also show, at the ECoG level, a distinct suppression of the slow oscillatory activity, something that has been reported to occur when intracortical connections are disrupted (Amzica and Steriade, 1995). Thus, it could be that in our case the cannabinoid agonist, which was administered intravenously to act upon endocannabinoid receptors, was actively disrupting either the intrinsic activity of the cortex, as has been previously reported in somatosensory and prefrontal areas (Auclair et al., 2000 and Fortin and Levine, 2007), or corticofugal glutamatergic neurotransmission as shown recently in basal ganglia/cortex interactions (Sales-Carbonell et al., 2013).

Within the thalamus, the control of the level of arousal is not simply linked to the level of excitatory inputs (brainstem or cortical) but must include the known inhibitory inputs, both from local, intrinsic GABAergic interneurons and the nearby GABAergic cells of the TRN, a network of interconnected inhibitory cells under the influence of the cortex that receive feedforward input from the thalamic cells they inhibit (Sherman and Guillery, 2002), a nucleus that is predominantly active during sleep-like states and that becomes depressed during conscious awareness. However the actual effect of the endocannabinoid system on the output from the TRN is puzzling since it could decrease the cortical input to the TRN reducing the inhibitory drive to relay cells (as shown in the somatosensory system by Sun et al., 2011), but it could also decrease lateral inhibitory connections inside the TRN potentially increasing the GABAergic input to relay cells (Sun et al., 2011).

Furthermore, it is well known that the effect of ACh on GABAergic cells, both intrinsic to the dLGN and resident in the TRN, is normally inhibitory (Godfraind, 1978 and McCormick and Pape, 1988). So, an endocannabinoid-mediated reduction in cholinergic drive could effectively enhance inhibition or alter the balance of interactions between inhibitory cells in unknown and complex ways. Furthermore, SalesCarbonell et al. (2013) have reported increased oscillatory activity in somatosensory cortex, which they ascribe to an increased inhibitory input to the thalamus, in their case arising from substantia nigra pars reticulata $(\mathrm{SNr})$, indicating that there may be a general link between cannabinoid control of thalamocortical oscillations which can be utilized by appropriately located inhibitory drives. In the case of the LGN, this would also be reflected in a measureable change in bursting activity

We can suggest several routes by which endocannainoids can alter the function of thalamic cells, both in simple terms of their firing rates and in terms of more complex oscillatory and bursting activities, but we cannot ascribe a single mechanism that is more likely than any another, which suggests that the likeliest is a combination of withdrawal of excitation, enhanced inhibition, and interactions within and between circuit elements. In future, new experiments -perhaps combining intracellular recordings and local drug application- could be used to further clarify the actual underlying mechanisms. However, the current study reveals the activation of thalamic CB1 receptors as a significant element involved in the regulation of thalamic rhythmicity, which has been postulated as a key element of sleep. Thus, these results also help us to understand the mechanisms mediating the known effect of cannabis as a sleepinducing agent.

\section{Conclusion}

The current study reveals for the first time that the activation of thalamic CB1 receptors by AEA is a significant element in regulating burst firing and oscillatory activity, two phenomena closely related to the transition between wake and sleep and the level of vigilance. We suggest that the endocannabinoid system is involved in regulating the level of alertness, including actions intrinsic to the sleep-wake cycle.

\section{Acknowledgements}

The work was supported by MICINN (BFU2009-08169) and Xunta de Galicia (Consellería de Educación-2007/000140-0), Spain. M. Dasilva was supported by the FPU program. We thank Dr. Nelson Espinosa for his technical assistance during experiments and data analysis. 


\section{References}

Acquas et al., 2000. E. Acquas, A. Pisanu, P. Marrocu, G. Di Chiara. Cannabinoid CB(1) receptor agonists increase rat cortical and hippocampal acetylcholine release in vivo. Eur $\mathbf{J}$ Pharmacol, 401 (2000), pp. 179-185

Amzica and Steriade, 1995. F. Amzica, M. Steriade. Disconnection of intracortical synaptic linkages disrupts synchronization of a slow oscillation. J Neurosci, 15 (1995), pp. 4658-4677

Auclair et al., 2000. N. Auclair, S. Otani, P. Soubrie, F. Crepel. Cannabinoids modulate synaptic strength and plasticity at glutamatergic synapses of rat prefrontal cortex pyramidal neurons. J Neurophysiol, 83 (2000), pp. 3287-3293

Buonamici et al., 1982. M. Buonamici, G.A. Young, N. Khazan. Effects of acute delta 9-THC administration on ECOG and ECOG power spectra in the rat. Neuropharmacology, 21 (1982), pp. 825-829

Consroe et al., 1997. P. Consroe, R. Musty, J. Rein, W. Tillery, R. Pertwee. The perceived effects of smoked cannabis on patients with multiple sclerosis. Eur Neurol, 38 (1997), pp. 44-48

Dasilva et al., 2012. M. Dasilva, K.L. Grieve, J. Cudeiro, C. Rivadulla. Endocannabinoid CB1 receptors modulate visual output from the thalamus. Psychopharmacology, 219 (2012), pp. 835-845

Devane et al., 1992. W.A. Devane, L. Hanus, A. Breuer, R.G. Pertwee, L.A. Stevenson, G. Griffin, D. Gibson, A. Mandelbaum, A. Etinger, R. Mechoulam. Isolation and structure of a brain constituent that binds to the cannabinoid receptor. Science, 258 (1992), pp. 1946-1949

Di Marzo et al., 1994. V. Di Marzo, A. Fontana, H. Cadas, S. Schinelli, G. Cimino, J.C. Schwartz, D. Piomelli. Formation and inactivation of endogenous cannabinoid anandamide in central neurons. Nature, 372 (1994), pp. 686-691

Dossi et al., 1992. R.C. Dossi, A. Nuñez, M. Steriade. Electrophysiology of a slow $(0.5-4 \mathrm{~Hz})$ intrinsic oscillation of cat thalamocortical neurones in vivo. J Physiol, 447 (1992), pp. 215-234

Ferraro et al., 2001. L. Ferraro, M.C. Tomasini, G.L. Gessa, B.W. Bebe, S. Tanganelli, T. Antonelli. The cannabinoid receptor agonist WIN 55,212-2 regulates glutamate transmission in rat cerebral cortex: an in vivo and in vitro study. Cereb Cortex, 11 (2001), pp. 728-733

Fortin and Levine, 2007. D.A. Fortin, E.S. Levine. Differential effects of endocannabinoids on glutamatergic and GABAergic inputs to layer 5 pyramidal neurons. Cereb Cortex, 17 (2007), pp. 163174

Freund et al., 2003. T.F. Freund, I. Katona, D. Piomelli. Role of endogenous cannabinoids in synaptic signaling. Physiol Rev, 83 (2003), pp. 1017-1066

Garey et al., 1991. L.J. Garey, B. Dreher, S.R. Robinson. The organization of the visual thalamus. B. Dreher, S.R. Robinson (Eds.), Neuroanatomy of the visual pathways and their development, Macmillan Press, Houndmills and London (1991)

Godfraind, 1978. J.M. Godfraind. Acetylcholine and somatically evoked inhibition on perigeniculate neurones in the cat. Br J Pharmacol, 63 (1978), pp. 295-302

Grieve et al., 2009. K.L. Grieve, C. Rivadulla, J. Cudeiro. Mixed burst and tonic firing in the thalamus: a study in the feline lateral geniculate nucleus in vivo. Brain Res, 1273 (2009), pp. 48-57

Guido et al., 1992. W. Guido, S.M. Lu, S.M. Sherman. Relative contributions of burst and tonic responses to the receptive field properties of lateral geniculate neurons in the cat. J Neurophysiol, 68 (1992), pp. 2199-2211

Hájos et al., 2000. N. Hájos, I. Katona, S.S. Naiem, K. MacKie, C. Ledent, I. Mody, T.F. Freund. Cannabinoids inhibit hippocampal GABAergic transmission and network oscillations. Eur J Neurosci, 12 (2000), pp. 3239-3249

Hajós et al., 2008. M. Hajós, W.E. Hoffmann, B. Kocsis. Activation of cannabinoid-1 receptors disrupts sensory gating and neuronal oscillation: relevance to schizophrenia. Biol Psychiatry, 63 (2008), pp. 1075-1083

Hughes et al., 2002. S.W. Hughes, D.W. Cope, K.L. Blethyn, V. Crunelli. Cellular mechanisms of the slow $(<1 \mathrm{~Hz})$ oscillation in thalamocortical neurons in vitro. Neuron, 33 (2002), pp. 947-958

Ilan et al., 2004. A.B. Ilan, M.E. Smith, A. Gevins. Effects of marijuana on neurophysiological signals of working and episodic memory. Psychopharmacology, 176 (2004), pp. 214-222

Jahnsen and Llinás, 1984. H. Jahnsen, R. Llinás. Voltage-dependent burst-to-tonic switching of thalamic cell activity: an in vitro study. Arch Ital Biol, 122 (1984), pp. 73-82

Kalil and Chase, 1970. R.E. Kalil, R. Chase. Corticofugal influence on activity of lateral geniculate neurons in the cat. J Neurophysiol, 33 (1970), pp. 459-474

Kim et al., 2002. J. Kim, M. Isokawa, C. Ledent, B.E. Alger. Activation of muscarinic acetylcholine receptors enhances the release of endogenous cannabinoids in the hippocampus. J Neurosci, 22 (2002), pp. 10182-10191 
Livingstone and Hubel, 1981. M.S. Livingstone, D.H. Hubel. Effects of sleep and arousal on the processing of visual information in the cat. Nature, 291 (1981), pp. 554-561

Lu et al., 1992. S.M. Lu, W. Guido, S.M. Sherman. Effects of membrane voltage on receptive-field properties of lateral geniculate neurons in the cat - contributions of the low-threshold ca-2+ conductance. J Neurophysiol, 68 (1992), pp. 2185-2198

Maejima et al., 2001. T. Maejima, K. Hashimoto, T. Yoshida, A. Aiba, M. Kano. Presynaptic inhibition caused by retrograde signal from metabotropic glutamate to cannabinoid receptors. Neuron, 31 (2001), pp. 463-475

Mason and Cheer, 2009. R. Mason, J.F. Cheer. Cannabinoid receptor activation reverses kainate-induced synchronized population burst firing in rat hippocampus. Front Integr Neurosci, 3 (2009), p. 13

Matsuda et al., 1990. L.A. Matsuda, S.J. Lolait, M.J. Brownstein, A.C. Young, T.I. Bonner. Structure of a cannabinoid receptor and functional expression of the cloned cDNA. Nature, 346 (1990), pp. 561-564

McCormick and Pape, 1988. D.A. McCormick, H.C. Pape. Acetylcholine inhibits identified interneurons in the cat lateral geniculate nucleus. Nature, 334 (1988), pp. 246-248

McCormick and von Krosigk, 1992. D.A. McCormick, M. von Krosigk. Corticothalamic activation modulates thalamic firing through glutamate "metabotropic" receptors. Proc Natl Acad Sci U S A, 89 (1992), pp. 2774-2778

Mechoulam et al., 1995. R. Mechoulam, S. Ben-Shabat, L. Hanus, M. Ligumsky, N.E. Kaminski, A.R. Schatz, et al. Identification of an endogenous 2-monoglyceride, present in canine gut, that binds to cannabinoid receptors. Biochem Pharmacol, 50 (1995), pp. 83-90

Morgan et al., 2008. N.H. Morgan, I.M. Stanford, G.L. Woodhall. Modulation of network oscillatory activity and GABAergic synaptic transmission by CB1 cannabinoid receptors in the rat medial entorhinal cortex. Neural Plast, 2008 (2008), p. 808564

Munro et al., 1993. S. Munro, K.L. Thomas, M. Abu-Shaar. Molecular characterization of a peripheral receptor for cannabinoids. Nature, 365 (1993), pp. 61-65

Murillo-Rodríguez et al., 1998. E. Murillo-Rodríguez, M. Sánchez-Alavez, L. Navarro, D. MartínezGonzález, R. Drucker-Colín, O. Prospéro-García. Anandamidemodulatessleep and memory in rats. Brain Res, 812 (1998), pp. 270-274

Murillo-Rodríguez et al., 2001. E. Murillo-Rodríguez, R. Cabeza, M. Méndez-Díaz, L. Navarro, O. Prospéro-García. Anandamide-induced sleep is blocked by SR141716A, a CB1 receptor antagonist and by U73122, a phospholipase C inhibitor. Neuroreport, 12 (2001), pp. 2131-2136

Paxinos and Watson, 2007. G. Paxinos, C. Watson. The rat brain in stereotaxic coordinates. Elsevier (2007)

Piomelli, 2003. D. Piomelli. The molecular logic of endocannabinoid signaling. Nat Rev Neurosci, 4 (2003), pp. 873-884

Robbe et al., 2006. D. Robbe, S.M. Montgomery, A. Thome, P.E. Rueda-Orozco, B.L. McNaughton, G. Buzsaki. Cannabinoids reveal importance of spike timing coordination in hippocampal function. Nat Neurosci, 9 (2006), pp. 1526-1533

Sales-Carbonell et al., 2013. C. Sales-Carbonell, P.E. Rueda-Orozco, E. Soria-Gómez, G. Buzsáki, G. Marsicano, D. Robbe. Striatal GABAergic and cortical glutamatergic neurons mediate contrasting effects of cannabinoids on cortical network synchrony. Proc Natl Acad Sci U S A, 110 (2013), pp. 719-724

Santucci et al., 1996. V. Santucci, J.J. Storme, P. Soubrié, G. Le Fur. Arousal-enhancing properties of the CB1 cannabinoid receptor antagonist SR 141716A in rats as assessed by electroencephalographic spectral and sleep-waking cycle analysis. Life Sci, 58 (1996), pp. 103-110

Schlicker and Kathmann, 2001. E. Schlicker, M. Kathmann. Modulation of transmitter release via presynaptic cannabinoid receptors. Trends Pharmacol Sci, 22 (2001), pp. 565-572

Sefton et al., 1981. A.J. Sefton, A. Mackay-Sim, L.A. Baur, L.J. Cottee. Cortical projections to visual centres in the rat: an HRP study. Brain Res, 215 (1981), pp. 1-13

Sherman and Guillery, 2002. S.M. Sherman, R.W. Guillery. The role of the thalamus in the flow of information to the cortex. Philos Trans R Soc Lond B Biol Sci, 357 (2002), pp. 1695-1708

Steriade, 2003. M. Steriade. Thecorticothalamic system in sleep. Front Biosci, 8 (2003), pp. 878-899

Steriade, 2004. M. Steriade. Acetylcholine systems and rhythmic activities during the waking-sleep cycle. Prog Brain Res, 145 (2004), pp. 179-196

Steriade et al., 1993. M. Steriade, D.A. McCormick, T.J. Sejnowski. Thalamocortical oscillations in the sleeping and aroused brain. Science, 262 (1993), pp. 679-685

Sugiura et al., 1996. T. Sugiura, S. Kondo, A. Sukagawa, T. Tonegawa, S. Nakane, A. Yamashita. Transacylase-mediated and phosphodiesterase-mediated synthesis of $\mathrm{N}$-arachidonoylethanolamine, an endogenous cannabinoid-receptor ligand, in rat brain microsomes. Comparison with synthesis from free arachidonic acid and ethanolamine. Eur J Biochem, 240 (1996), pp. 53-62 
Sun et al., 2011. Y.G. Sun, C.S. Wu, H.C. Lu, M. Beierlein. Target-dependent control of synaptic inhibition by endocannabinoids in the thalamus. J Neurosci, 31 (2011), pp. 9222-9230

Timofeev and Steriade, 1996. I. Timofeev, M. Steriade. Low-frequency rhythms in the thalamus of inctact-cortex and decorticated cats. J Neurophysiol, 76 (1996), pp. 4152-4168

Waleszczyk et al., 2005. W.J. Waleszczyk, M. Bekisz, A. Wróbel. Cortical modulation of neuronal activity in the cat's lateral geniculate and perigeniculate nuclei. Exp Neurol, 196 (2005), pp. 54-72

Willinsky et al., 1975. M.D. Willinsky, A. Loizzo, V.G. Longo. ECOG spectral analysis for the evaluation of the central effects of delta6-tetrahydrocannabinol in rabbits. Psychopharmacologia, 41 (1975), pp. 123-126 